\title{
Colon Ischaemia Induced by Amoxicillin-related Anaphylactic Shock
}

\author{
Nuno Melo ${ }^{1}$, Isabel Hipólito ${ }^{2}$, Joana Alves Pereira ${ }^{1}$, Pedro Cunha ${ }^{2}$, Jorge Almeida ${ }^{1}$ \\ ${ }^{1}$ Internal Medicine Department, Centro Hospitalar e Universitário de São João, Porto, Portugal \\ ${ }^{2}$ Intensive Care Department, Centro Hospitalar e Universitário de São João, Porto, Portugal
}

Received: $14 / 05 / 2021$

Accepted: 30/05/2021

Published: 10/06/2021

How to cite this article: Melo N, Hipólito I, Alves Pereira J, Cunha P, Almeida J. Colon iscaemia induced by amoxicillin-related anaphylactic shock. EJCRIM 2021;8: doi:10.12890/2021_002640.

Conflicts of Interests: The authors declare there are no competing interests.

This article is licensed under a Commons Attribution Non-Commercial 4.0 License

\section{ABSTRACT}

Colon ischaemia is the most frequent type of ischaemia of the digestive tract. It is more common among the elderly, especially patients with haemodynamic disturbance. In young patients, it is associated with particular drugs such as oral contraceptives and ergotamine derivatives. Anaphylaxis is characterized by acute onset and skin and mucosal involvement together with cardiovascular involvement. Gastrointestinal symptoms are common in anaphylaxis, but gastrointestinal bleeding and acute colon ischaemia are rarely described. Here, we present the case of a 52-year-old woman with acute ischaemic colitis associated with an anaphylactic reaction to amoxicillin.

\section{LEARNING POINTS}

- In young patients, drugs may play a role in colon ischaemia through several different mechanisms, including anaphylaxis.

- Although gastrointestinal symptoms are common in anaphylaxis, colon ischaemia is rare in this context.

- The exact mechanism of colon ischaemia in anaphylaxis is not known, but gut hypoperfusion secondary to hypotension may play a role.

\section{KEYWORDS}

Colon ischemia, anaphylaxis, amoxicillin

\section{INTRODUCTION}

Colon ischaemia is a common type of ischaemic insult, resulting from decreased arterial blood flow to the colon ${ }^{[1]}$. It is more frequent among the elderly and a recently published population-based study yielded an incidence of 16.3 cases/100,000 person-years, with annual incidence rates ranging from 1.1 per 100,000 among those under age 40 years to 107 per 100,000 among those aged 80 years or older ${ }^{[2]}$. Among older patients, it is usually associated with heart failure, myocardial infarction, acute haemorrhage, septic shock, hypotension or dehydration. On the other hand, risk factors in young adults are not clearly established, but extreme exercise and some drugs such as oral contraceptives or alosetron seem to increase the risk of colon ischaemia in this population ${ }^{[3]}$.

Amoxicillin is one of most frequently prescribed beta-lactams and is often associated with allergic reactions. In a recent study, penicillins were the drug class most commonly associated with anaphylaxis, with a prevalence of 45.9 per 10,000 patients, and amoxicillin was the most common causative drug ${ }^{[4]}$. Colon ischaemia induced by anaphylactic shock in the context of antibiotherapy has been rarely described ${ }^{[5-7]}$. The authors present the case of a patient with colon ischaemia secondary to anaphylactic shock due to amoxicillin. 


\section{CASE DESCRIPTION}

This case report describes a 52-year-old woman with a smoking habit, no prior medical history and not on chronic medication. She had no personal or familial history of allergy or atopy.

She was admitted to the emergency room due to an episode of non-bloody vomit followed by syncope, which occurred some minutes after she took amoxicillin $1 \mathrm{~g}$ prescribed for a dental infection.

At admission, she was confused (Glasgow Coma Scale score of 11) and hypotensive (blood pressure (BP) of $65 / 40 \mathrm{mmHg}$ ) with no other relevant findings on physical examination, such as cutaneous signs of allergy or respiratory distress. Intravenous fluid therapy with $0.9 \%$ sodium chloride and $200 \mathrm{mg}$ of intravenous hydrocortisone were administered.

The patient recovered her mental and haemodynamic status (BP 120/81 mmHg) after 2 litres of intravenous 0.9\% sodium chloride were administered. Blood analyses showed high haemoglobin $(18.1 \mathrm{~g} / \mathrm{dl})$, leucocytosis (leucocytes 26,000 cells $/ \mathrm{mm}^{2}$, with normal eosinophil count) and mild acute kidney injury (urea/creatinine 50/1.1 mg/dl) with metabolic acidaemia $\left(\mathrm{pH} 7.31, \mathrm{pO}_{2} 82.3 \mathrm{mmHg}, \mathrm{pCO}_{2} 37.4 \mathrm{mmHg}\right.$, $\mathrm{HCO}_{3}-18.5 \mathrm{mmol} / \mathrm{l}$, lactate $1.95 \mathrm{mmol} / \mathrm{I}$ (normal range $\left.0.5-1 \mathrm{mmol} / \mathrm{l}\right)$ ). C-reactive protein was low (18.8 mg/l) and coagulation tests were normal.

A couple of hours after admission, the patient developed acute lower abdominal pain followed by rectal bleeding with no haemodynamic instability. Rectal sigmoidoscopy was performed, which showed diffuse erythema with friability and a segment with a vinous aspect suggestive of necrosis $11 \mathrm{~cm}$ from the anal margin (Fig. 1).

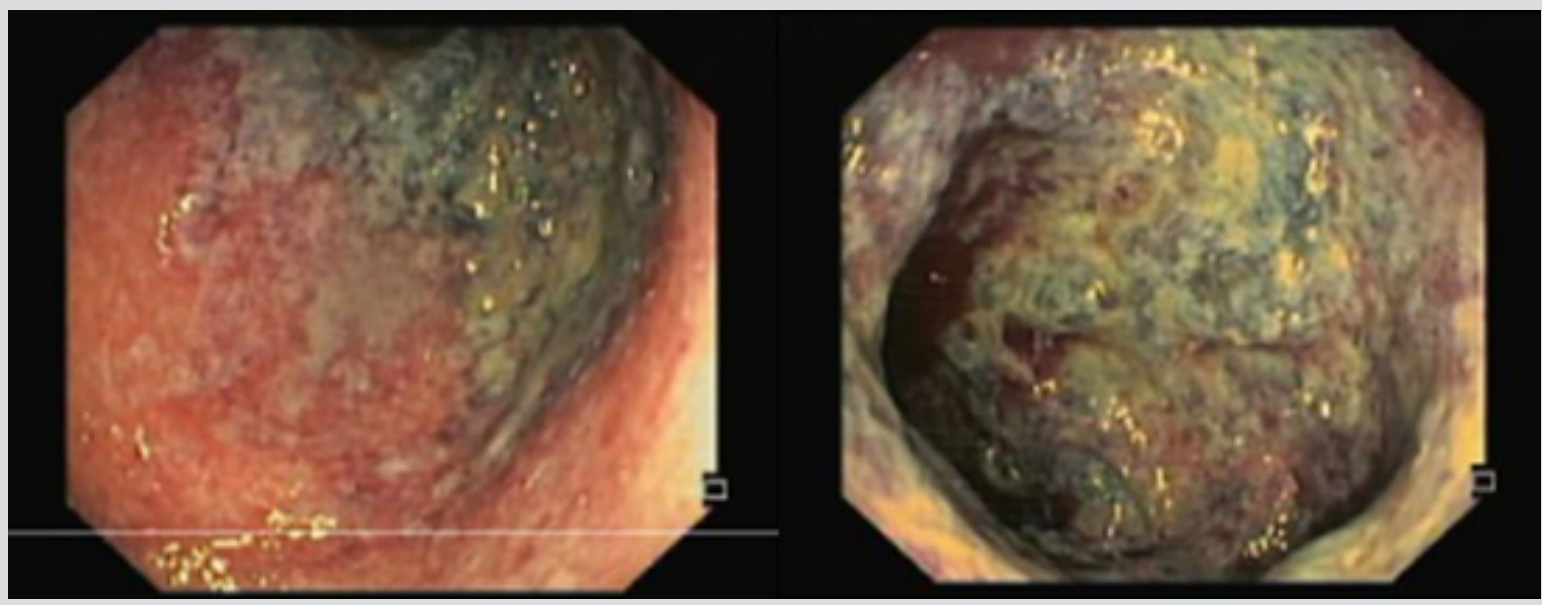

Figure 1. Diffuse mucosal erythema and some areas with a vinous aspect suggestive of mucosal necrosis

Her electrocardiogram showed sinus rhythm. Further evaluation with computed tomography angiography excluded filling defects in the aorta and its branches, but showed diffuse colonic mucosal oedema, suggestive of proctocolitis. She was admitted for further observation. Food was slowly re-introduced. No further blood losses were registered during her hospital stay. Microbiological studies were negative. An autoimmune panel (anti-nuclear antibodies, anti-neutrophil cytoplasmic antibodies, anticardiolipin and lupus anticoagulant) was also negative. Gut biopsies revealed structural changes compatible with colon ischaemia. Ultimately, the diagnosis of acute colon ischaemia secondary to anaphylactic reaction was presumed and the patient was discharged with advice to avoid amoxicillin and its derivatives. Six weeks later, serum specific IgE antibody levels against amoxicillin, penicillin $\mathrm{G}$ and penicillin $\mathrm{V}$ were determined and a positive result was obtained against amoxicillin, which supports the diagnosis. The patient was lost to follow-up and further diagnostic evaluation was not carried out.

\section{DISCUSSION}

The differential diagnosis of haematochezia with contiguous rectal colitis includes infectious, inflammatory and ischaemic aetiologies. In our patient, the diagnosis of antibiotic-associated colitis could be considered, but antibiotic exposure was too short (only one dose) as was the time between antibiotic exposure and symptom development; moreover, the clinical evolution was very different from that observed in this clinical entity. The clinical course and the colonoscopic and histological features of our patient were typical of colon ischaemia.

Colon ischaemia is the most common manifestation of mesenteric ischaemia, being more common among elderly patients with several 
comorbid conditions such as hypertension, diabetes mellitus, vascular disease, chronic kidney disease, cardiac or vascular surgery, or a hypercoagulable state ${ }^{[1]}$. On the other hand, the presence of colon ischaemia in young patients should always raise the possibility of drug involvement, especially oral contraceptives, and migraine drugs, like ergotamine derivatives, which are associated with hypercoagulable states and focal vasospasm, respectively ${ }^{[3]}$.

Intestinal ischaemia develops as a result of three mechanisms: non-occlusive colonic ischaemia, embolic thrombotic arterial occlusion, and mesenteric venous thrombosis. The colon is the region of the gastrointestinal tract most affected as a result of hypoperfusion. In addition, the colonic wall is thicker than the small intestine wall and contains fewer microvascular plexuses.

Anaphylaxis is considered in patients who present an acute onset illness with typical skin features (urticarial rash or erythema/flushing, and/ or angio-oedema) and involvement of respiratory and/or cardiovascular and/or persistent severe gastrointestinal symptoms ${ }^{[8]}$. Our patient did not present skin symptoms, but cutaneous symptoms are absent in around $10 \%$ of anaphylactic reactions ${ }^{[8]}$. Anaphylaxis is known to cause several gastroenterological symptoms - most commonly vomiting and diarrhoea - but rare intestinal bleeding from non-occlusive mesenteric ischaemia has been reported ${ }^{[9]}$. In our patient, transient hypotension could have caused gut hypoperfusion and subsequent colon ischaemia. In fact, the rectosigmoid junction is a specific area known to be vulnerable to ischaemic lesions due to limited connections with the main intestinal arteries. There was no histological evidence of direct or immune-induced gut damage. There was no clinical evidence of any other cause of colon ischaemia, such as alternative drugs or comorbid conditions, except the amoxicillin allergy and anaphylactic shock in this patient.

Although very rare, there are a few reports of anaphylaxis and colon ischaemia. To date only one case associates amoxicillin with colon ischaemia ${ }^{[5]}$.

This case highlights the importance of drug review in young patients with colon ischaemia, since drugs may be associated with colon ischaemia through several mechanisms, including anaphylaxis. Although rare, colon ischaemia may occur several hours after anaphylaxis and thus, vigilance for blood loss is warranted. Apart from anaphylaxis treatment, patients should be advised for drug eviction and referred to allergy units for desensitization and further evaluation.

\section{REFERENCES}

1. Misiakos EP, Tsapralis D, Karatzas T, Lidoriki I, Schizas D, Sfyroeras GS, et al. Advents in the diagnosis and management of ischemic colitis. Front Surg 2017;4:47.

2. Yadav S, Dave M, Edakkanambeth Varayil J, Harmsen WS, Tremaine WJ, Zinsmeister AR, et al. A population-based study of incidence, risk factors, clinical spectrum, and outcomes of ischemic colitis. Clin Gastroenterol Hepatol 2015;13(4):731-738.e6.

3. Brandt LJ, Feuerstadt P, Longstreth GF, Boley SJ. ACG Clinical Guideline: epidemiology, risk factors, patterns of presentation, diagnosis, and management of colon ischemia (Cl). Am J Gastroenterol 2015;110(1):18-44.

4. Dhopeshwarkar N, Sheikh A, Doan R, Topaz M, Bates DW, Blumenthal KG, et al. Drug-induced anaphylaxis documented in electronic health records. J Allergy Clin Immunol Pract 2019:7(1):103-111.

5. Perez-Carral C. Acute ischaemic colitis due to hypotension and amoxicillin allergy. Postgrad Med J 2004;80(943):298-299.

6. Alonge M, Benini F, Cannatelli R, Pozzi A, Missale G, Villanacci V, et al. Amoxicillin-clavulanate-induced ischaemic colitis. Case Rep Gastroenterol 2020;14(1):242-247.

7. Gül Utku Ö, Ergül B, Balci M, Oğuz D. A rare case of ischemic colitis: cefuroxime-related anaphylactic shock. Gastroenterol Nurs 2019;42(5):448-450.

8. Anagnostou K, Turner PJ. Myths, facts and controversies in the diagnosis and management of anaphylaxis. Arch Dis Child 2019;104(1):83-90.

9. Park S, Chun H, Keum B, Seo Y, Kim Y, Jeen Y, et al. Anaphylactic shock-induced ischemic proctocolitis following bee stings: first case report. Endoscopy 2010;42(S 02):E153154. 